\title{
Three-Probe Error Separation with Chromatic Confocal Sensors for Roundness Measurement
}

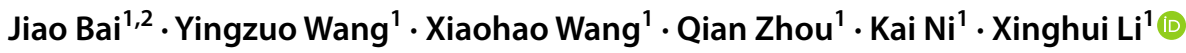

Received: 6 July 2021 / Revised: 18 October 2021 / Accepted: 19 October 2021 / Published online: 15 November 2021

(c) The Author(s) 2021

\begin{abstract}
In this study, three-probe error separation was developed with three chromatic confocal displacement sensors for roundness measurement. Here, the harmonic suppression is discussed first to set suitable orientation angles among three sensors. Monte Carlo simulation is utilized to test the error separation and optimize the orientation angles and off-axial distance. The experimental setup is established using chromatic confocal sensors with a precise rotary platform. The experimental results show that the measured roundness with an orientation-angle combination of $\left(0^{\circ}, 90.1^{\circ}\right.$, and $\left.178.6^{\circ}\right)$ is much better than that of another nonoptimal selection $\left(0^{\circ}, 90.4^{\circ}\right.$, and $\left.177.4^{\circ}\right)$. The roundness error is only $0.7 \%$ between the proposed measurement system and an expensive ultraprecision roundness meter. Furthermore, it is proven that the eccentricity distance should be decreased as small as possible to improve the measurement accuracy. In sum, this paper proposes a feasible method for roundness measurement with reliable simulations, easily integrated sensors, and an ordinary precision rotary platform.
\end{abstract}

Keywords Roundness measurement · Three-probe error separation · Chromatic confocal sensor · Monte Carlo simulation

\section{Introduction}

Roundness is one of the most important geometrical specifications of rotational workpieces for product-quality estimation, in situ processing optimization, and spindle accuracy test $[1,2]$. As the manufacturing accuracy is rapidly developed using precise or ultraprecise spindles, the roundness measurement is urgently required to provide micrometerlevel or even nanometer-level accuracy.

At present, many methods have been proposed to determine the roundness error. The most common method is to use a coordinate measuring machine or precise scan line stage to reconstruct a workpiece's profile for its roundness [3-5]. The measurement accuracy is seriously affected by the scan path, scan speed, and compensation algorithm of the contacting probe.

Jiao Bai and Yingzuo Wang contributed equally to this work.

Xinghui Li

li.xinghui@sz.tsinghua.edu.cn

1 Shenzhen International Graduate School, Tsinghua University, Shenzhen 518055, China

2 Institute of Materials, China Academy of Engineering Physics, Mianyang 621907, China
The single-probe method is another simple and direct method with a displacement probe and high-precision rotary platform $[6,7]$. Except for the accuracy of the probe, the rotation error of the rotary platform should be as small as possible to ensure that all measured displacements begin from the rotation axis. In this way, the profile of the workpiece is easily reconstructed with radial displacement. Thus, roundness can be derived from the profile using the least squares circle or other algorithms [8-10]. However, the high-precision rotary platform is usually expensive, and the eccentricity error of the displacement sensor seriously affects the measurement accuracy.

To acquire precise roundness using an ordinary rotation platform, the error separation technique was proposed to separate the artifact roundness and rotation error [11-15]. In the error separation technique, the reversal method and multiprobe method are most commonly used. The former assumes that the rotation error is repeatable and the reverse adjustment of the displacement probe or workpiece needs to be operated for every measurement [12, 16-18]. Although the separation accuracy is relatively higher than that of other methods, it is difficult to guarantee a complex fixture, accurate reverse angle of $180^{\circ}$, and good data synchronization.

The multiprobe method is widely used for measuring the in situ spindle error or workpiece roundness error, with three 
or more displacement sensors [12, 19-21]. These probes are fixed at permanent locations, and the workpiece is rotated by the rotary platform, improving the measurement flexibility and efficiency. Of course, more probes would cause more difficulty in the adjustment of the measurement system, and the uncertainty analysis is complex with various error sources, such as studies on the four-probe method [22].

Typically, the three-probe method is relatively simple to realize and can decode most components of the profile using three synchronous displacement sensors [21, 23-27]. Because the signal process involves the division operation in the frequency domain, harmonic suppression usually happens for some harmonic orders. Further studies found that the harmonic suppression mainly depends on the greatest common divisor of two orientation angles and $180^{\circ}$, allowing for many flexible choices. Kato et al. [23] proposed a typical orientation-angle combination, i.e., $\left(0^{\circ}, 38^{\circ}\right.$, and $67^{\circ}$ ), to decrease the influence of harmonic suppression. Cappa et al. [24] and Ding et al. [21] reduced the suppression of low-order harmonics through optimization of measurement angles. Moreover, Gao and Kiyono [25] combined the generalized three-point method and sequential three-point method to improve the robustness in stepwise variations. Shi et al. [26] proposed a hybrid three-probe method using two different mathematical operations to optimize individual harmonic coefficients. Chen et al. [27] introduced a solving system of the multivariable equation method to simplify the mathematical calculation process.

Furthermore, there are many noncontact choices of the displacement sensors for the three-probe method, such as capacitance sensors [21, 24, 25] and optical sensors [20, 26]. These sensors provide accuracy displacement during a rotation without contacting the rugged surface of the workpiece and are more stable and safer than contact probes. Thus, noncontact sensors have been increasingly used because of their high precision, sensitivity, and flexibility. However, the valid spot sizes on the workpiece surface of capacitance sensors or triangulation laser sensors are always as big as a millimeter lever. Clearly, this condition will seriously affect the lateral resolution in the profile reconstruction, which may cause a certain roundness measurement error, especially for workpieces with a small radius.

Accordingly, to solve the above-mentioned problem, in this study, we employed chromatic confocal sensors with tens of micrometer spot size and submicron longitudinal resolution. Chromatic confocal sensors take advantage of the dispersion of white light and the confocal technique to encode axial positions with the wavelength of the reflected light from the measured surface [28-31]. The dispersion probe focuses the light on the measured surface to produce a very small spot size, similar to that in a microscope objective. Moreover, the displacement only depends on the wavelength of the focus light, allowing for a relatively large tilt error to reflect little light to the conjugate detection pinhole. This advantage makes its measured displacement reliable and sensitive in the axial direction for curved surfaces [32, 33]. Thus, we believe that chromatic confocal sensors can be used in the three-probe method to improve measurement accuracy.

In this study, we first explored the effect of the orientation-angle combination and eccentricity distance and then acquired an optimized combination through the Monte Carlo simulation. Next, an experimental setup was built up for the roundness measurement of a cylindrical workpiece with three similar chromatic confocal sensors. Lastly, we explored the actual influences of the orientation-angle combination and off-axial distance on the roundness measurement.

\section{Principles}

\subsection{Three-Probe Method}

Three similar displacement sensors were mounted around the rotary platform, on which the workpiece is fixed, as shown in Fig. $1 . O$ is the rotation center, and $P$ is the workpiece center. Thus, the eccentricity distance $|O P|$ is constant during the rotation. The orientation angle between Probe $_{\mathrm{A}}$ and Probe $_{\mathrm{B}}$ is $\alpha$, whereas that between Probe $\mathrm{A}_{\mathrm{A}}$ and Probe $\mathrm{C}_{\mathrm{C}}$ is $\beta$, which is positive in the clockwise direction. Then, three continuous displacement signals $\left[S_{\mathrm{A}}(\theta), S_{\mathrm{B}}(\theta)\right.$, and $\left.S_{\mathrm{C}}(\theta)\right]$ were obtained synchronously at every rotation angle of $\theta$.

To clearly express the dynamic change of the three signals, we used $r(\theta)$ as the radius of the workpiece at the rotation angle $\theta$. Moreover, the rotation error of the rotary platform $\delta(\theta)$ was divided as $\delta_{x}(\theta)$ and $\delta_{y}(\theta)$ at the $x$-axis and $y$-axis. Then, the output signals were determined as below when the eccentricity distance $|O P|$ is as small as possible. In actual scenarios, we recommend that the maximum tolerable $|O P|$ should be smaller than the rotation error to avoid its influence in Eq. (1).

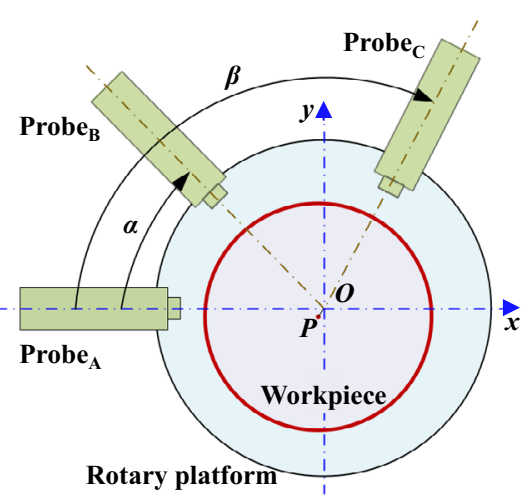

Fig. 1 Schematic diagram of the three-probe method 


$$
\left\{\begin{array}{l}
S_{\mathrm{A}}(\theta)=r(\theta)+\delta_{x}(\theta) \\
S_{\mathrm{B}}(\theta)=r(\theta+\alpha)+\delta_{x}(\theta) \cdot \cos \alpha+\delta_{y}(\theta) \cdot \sin \alpha \\
S_{\mathrm{C}}(\theta)=r(\theta+\beta)+\delta_{x}(\theta) \cdot \cos \beta+\delta_{y}(\theta) \cdot \sin \beta
\end{array}\right.
$$

It is difficult to extract the profile of the workpiece $r(\theta)$ from three known signals using ordinary algorithms. Thus, the error separation technique becomes a useful method to remove the unknown rotation error in the frequency domain. In detail, three signals were multiplied by three different coefficients $\left(c_{0}, c_{1}\right.$, and $\left.c_{2}\right)$ to be added together as a synthetic signal $S(\theta)$.

$$
\begin{aligned}
S(\theta)= & c_{0} S_{\mathrm{A}}(\theta)+c_{1} S_{\mathrm{B}}(\theta)+c_{2} S_{\mathrm{C}}(\theta) \\
= & c_{0}\left[r(\theta)+\delta_{x}(\theta)\right]+c_{1}\left[r(\theta+\alpha)+\delta_{x}(\theta) \cdot \cos \alpha+\delta_{y}(\theta) \cdot \sin \alpha\right] \\
& +c_{2}\left[r(\theta+\beta)+\delta_{x}(\theta) \cdot \cos \beta+\delta_{y}(\theta) \cdot \sin \beta\right]
\end{aligned}
$$

Through the simple deformation of $S(\theta)$, we can separate the profile function $r(\theta)$ and rotation error functions $\left[\delta_{x}(\theta)\right.$, $\left.\delta_{y}(\theta)\right]$.

$S(\theta)=c_{0} r(\theta)+c_{1} r(\theta+\alpha)+c_{2} r(\theta+\beta)$

$+\left(c_{0}+c_{1} \cos \alpha+c_{2} \cos \beta\right) \cdot \delta_{x}(\theta)+\left(c_{1} \sin \alpha+c_{2} \sin \beta\right) \cdot \delta_{y}(\theta)$

If the coefficients of $\delta_{x}(\theta)$ and $\delta_{y}(\theta)$ are set zeros, then we can get two equations for $c_{0}, c_{1}$, and $c_{2}$.

$\left\{\begin{array}{l}c_{0}+c_{1} \cos \alpha+c_{2} \cos \beta=0 \\ c_{1} \sin \alpha+c_{2} \sin \beta=0\end{array}\right.$

In general, we can set $c_{0}$ as 1 to decompose the equation to obtain the other parameters $c_{1}$ and $c_{2}$ as follows:

$c_{0}=1, c_{1}=-\frac{\sin \beta}{\sin (\beta-\alpha)}, c_{2}=\frac{\sin \alpha}{\sin (\beta-\alpha)}$

At the same time, we can derive the expression of $S(\theta)$ :

$S(\theta)=c_{0} r(\theta)+c_{1} r(\theta+\alpha)+c_{2} r(\theta+\beta)$

Using the linear phase property of the Fourier transform, we can simplify the above formula as follows. $R(\omega)$ is the Fourier transmission of the profile $r(\theta)$, and $H(\omega)$ is the frequency-weight function.

$$
\begin{aligned}
& S(\omega)=c_{0} R(\omega)+c_{1} R(\omega) \mathrm{e}^{\mathrm{i} \omega \alpha}+c_{2} R(\omega) \mathrm{e}^{\mathrm{i} \omega \beta} \\
& =\left[c_{0}+c_{1} \mathrm{e}^{\mathrm{i} \omega \alpha}+c_{2} \mathrm{e}^{\mathrm{i} \omega \beta}\right] R(\omega)=H(\omega) R(\omega)
\end{aligned}
$$

Thus, we derive $R(\omega)$ when $H(\omega)$ is not zero. Then, the profile $r(\theta)$ is calculated using the inverse Fourier transform of $R(\omega)$.

$$
R(\omega)=\frac{S(\omega)}{H(\omega)}
$$

\subsection{Harmonic Suppression}

The error separation technique skillfully derives the profile $r(\theta)$ using the Fourier and inverse Fourier transforms. However, the frequency-weight function $H(\omega)$ is not always zero at every value of $\omega$, which causes harmonic suppression. As a result, some frequency components of the profile cannot be reconstructed in the error separation. Here, we translate the expression of $H(\omega)$ in plural form as follows:

$$
\begin{aligned}
& H(\omega)=c_{0}+c_{1}(\cos \omega \alpha+\mathrm{i} \sin \omega \alpha)+c_{2}(\cos \omega \beta+\mathrm{i} \sin \omega \beta) \\
& =c_{0}+c_{1} \cos \omega \alpha+c_{2} \cos \omega \beta+\mathrm{i}\left(c_{1} \sin \omega \alpha+c_{2} \sin \omega \beta\right)
\end{aligned}
$$

Thus, we can derive the following equations to make $H(\omega)=0$.

$\left\{\begin{array}{l}c_{0}+c_{1} \cos \omega \alpha+c_{2} \cos \omega \beta=0 \\ c_{1} \sin \omega \alpha+c_{2} \sin \omega \beta=0\end{array}\right.$

When $\omega=1$, Eq. (10) is the same as Eq. (4). Hence, 1 is definitely one of the harmonic suppression orders $\left(\omega_{s}\right)$. In addition to 1, there are other solutions to satisfy Eqs. (4) and (10). Based on the conditions of $c_{1} \neq 0, c_{2} \neq 0$, and $\alpha<\beta$, the coefficients $c_{0}, c_{1}$, and $c_{2}$ can be removed using Eqs. (4) and (10).

$\tan \frac{\left(\omega_{s}+1\right) \alpha}{2}=\tan \frac{\left(\omega_{s}+1\right) \beta}{2}$

Because of the periodicity and monotonicity of the tangent function, we can determine the unknown harmonic suppression order $\omega_{s}$ as follows:

$\omega_{s}=1$, or

$\omega_{s}=\frac{360}{\beta-\alpha} k-1, k, \omega_{s} \in N^{*}$

It is concluded that the harmonic suppression is mainly caused by the orientation angles, which should be chosen properly in the three-probe method. $H\left(\omega_{s}\right)$ is usually set as 1 to take part in the division of Eq. (8), and then $R\left(\omega_{s}\right)$ is set as 0 to calculate $r(\theta)$ using the inverse Fourier transform. Because the high-frequency components of $R\left(\omega_{s}\right)$ are always small to be ignored, $\omega_{s}$ should be as big as possible except for $\omega_{s}=1$.

\section{Simulation}

In previous studies, a typical orientation-angle combination was proposed, i.e., $\left(0^{\circ}, 38^{\circ}\right.$, and $\left.67^{\circ}\right)$. However, these angles are a little dense to make sensors close with one another, which may easily cause interferences when measuring thin workpieces. To obtain a suitable orientation-angle combination with big intervals, the Monte Carlo simulation was 
performed to explore the influence of orientation angles on the error separation accuracy of the cylindrical workpiece.

Based on the experimental experience, the frequency components of the rotation error $\delta(\theta)$ and profile $r(\theta)$ at $\omega>50$ are both relatively small enough to affect the measurement results. Hence, we designed two types of frequency spectra for the rotation error and profile. Their amplitude distribution spectra ranges selected randomly between the upper and lower curves are shown in Fig. 2a, b. The phase distributions were all randomly selected from $0^{\circ}$ to $360^{\circ}$. In Fig. 2c, e, two rotation error curves are randomly designed using the inverse fast Fourier transform according to the randomly selected frequency spectrum. Moreover, we plot two random profiles with a roundness of 4.45 and $4.69 \mu \mathrm{m}$ in Fig. 2d, f.

Except for the profile $r(\theta)$ and rotation error $\delta(\theta)$, the orientation-angle combination and eccentricity distance are also necessary for error separation. As the eccentricity distance is constant when the workpiece is fixed on the rotary platform, we allowed it to change, for example, from 0 to $25 \mu \mathrm{m}$. The orientation angles have a crucial influence on the harmonic suppression, so we set $\alpha$ randomly from $85^{\circ}$ to $95^{\circ}$ and $\beta$ from $175^{\circ}$ to $185^{\circ}$. The orientation-angle ranges provide a loose arrangement of three probes to avoid the interference caused by the big probe diameter but small working distance.
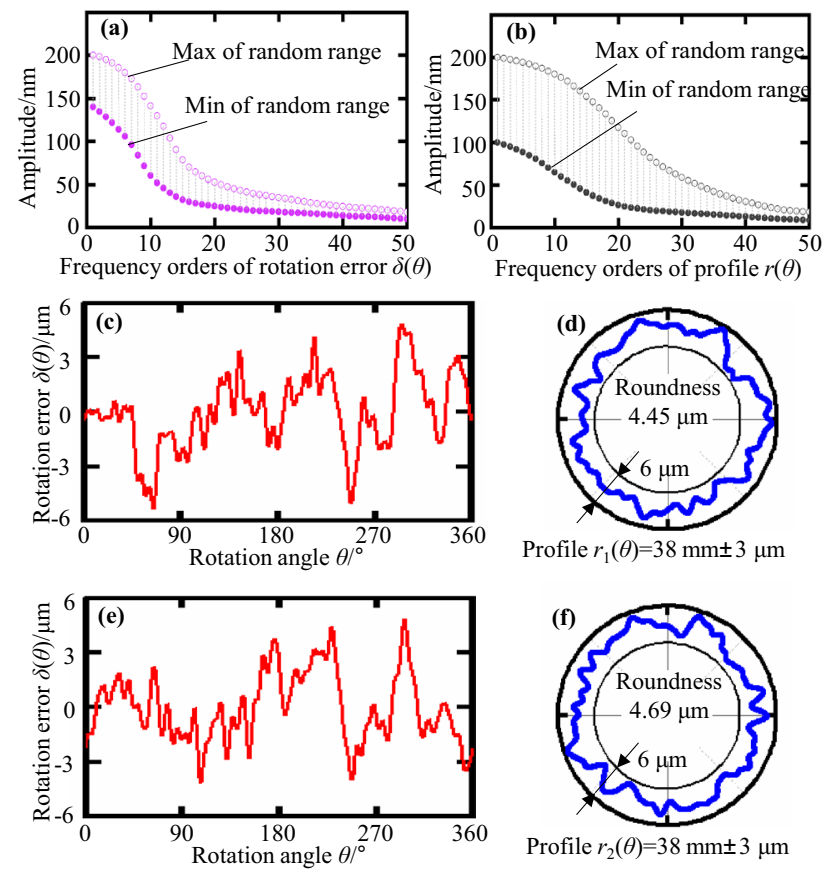

Fig. 2 Random design of the rotation error and profile: (a) amplitude distribution spectra range for rotation error; (b) amplitude distribution spectra range for profile; (c, e) two random rotation error curves; (d, f) two random profiles
With these orientation angles, the harmonic suppression orders $\omega_{s}$ are determined based on Eq. (12). In other words, the frequency components at $\omega_{s}$ of the profile $r(\theta)$ and rotation error $\delta(\theta)$ cannot be derived from the error separation calculation. In fact, the irregular direct current component of the profile at $\omega=0$ does not affect the calculation of roundness. Moreover, the eccentricity distance and linear component of the profile at $\omega_{s}=1$ always mix together to be ignored in the error separation technique and single-probe method. In brief, the three-probe method can reconstruct the profile at $\omega \geq 2$ except the other harmonic suppression orders $\omega_{s}$ in Eq. (12).

To determine the orientation angles $\alpha$ and $\beta$, we considered all the combinations among the ranges of $\left(85^{\circ}, 95^{\circ}\right)$ and $\left(175^{\circ}, 185^{\circ}\right)$ with an interval of $0.1^{\circ}$, except for $\beta=180^{\circ}$. Using the error separation technique, the roundness was derived from the reconstructed profile using the least squares method. In Fig. 3a, the roundness errors from the designed roundness are plotted with different orientation-angle combinations based on the Monte Carlo simulation approach. The results show that when the orientation angle $\beta$ changes within $2^{\circ}$ away from $180^{\circ}$, the roundness error varies little with a different orientation angle $\alpha$. Moreover, we explored the various suitable ranges of $\beta$ under 16 random sets of profiles and rotation errors, as shown in Fig. 3b. Based on a comparison, the optimized angle range of $\beta$ should be $178.4-179^{\circ}$, whereas the orientation angle $\alpha$ can be freely set as $85-95^{\circ}$.

Furthermore, we tested the profile reconstruction accuracy using error separation. Without loss of generality, the orientation-angle combination was designed as $\left(0^{\circ}, 90.1^{\circ}\right.$, and $\left.178.6^{\circ}\right)$, with other two combinations of $\left(0^{\circ}, 38^{\circ}\right.$, and
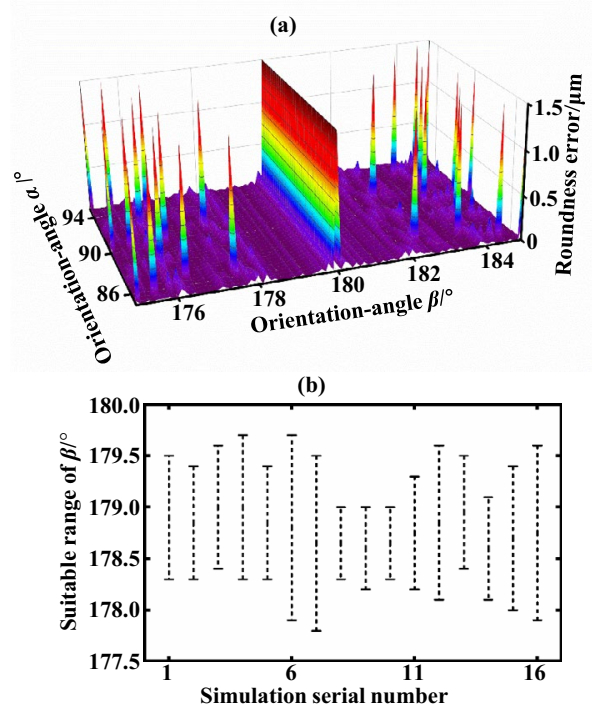

Fig. 3 a Roundness error at different orientation angles; b suitable ranges of $\beta$ in 16 simulations 
$\left.67^{\circ}\right)$ and $\left(0^{\circ}, 90.4^{\circ}\right.$, and $\left.177.4^{\circ}\right)$ for comparison. In the simulation, the profile and rotation error were set as those presented in Fig. 2b, c and Fig. 2e, f. The simulation results for the reconstructed profiles are shown in Fig. 4. Clearly, the profile reconstruction error is the biggest in the combination of $\left(0^{\circ}, 90.4^{\circ}\right.$, and $\left.177.4^{\circ}\right)$, whereas the other two can achieve an approximate reconstruction accuracy of approximately $\pm 20 \mathrm{~nm}$. In conclusion, the proposed combination of $\left(0^{\circ}, 90.1^{\circ}\right.$, and $\left.178.6^{\circ}\right)$ is suitable for the three-probe error separation as the traditional selection of $\left(0^{\circ}, 38^{\circ}\right.$, and $\left.67^{\circ}\right)$.

Lastly, the influence of the eccentricity distance $|O P|$ was explored in the Monte Carlo simulation. Similarly, the profile and rotation error were set as those presented in Fig. 2b, c and Fig. 2e, f. The orientation-angle combination is defined as $\left(0^{\circ}, 90.1^{\circ}\right.$, and $\left.178.6^{\circ}\right)$. The interval was set by $10 \mu \mathrm{m}$ from 10 to $190 \mu \mathrm{m}$. Then, the roundness of the reconstructed profile was determined and compared with the theoretical roundness. The roundness error varies with the eccentricity distance, as shown in Fig. 5. The results show that the roundness error increases when the eccentricity distance gets bigger. This trend is in accordance with our general experience that decreasing the eccentricity distance as small as possible improves the measurement accuracy.

\section{Experiment}

The basic principle sketch of the chromatic confocal sensor is plotted in Fig. 6a, b. The white light is dispersed and focused on the measured surface and then reflected to the spectrometer to derive its focus wavelength. The relationship between the focus wavelength and axial positions is named as the response curve, from which the corresponding position can be quickly derived with the focus wavelength.
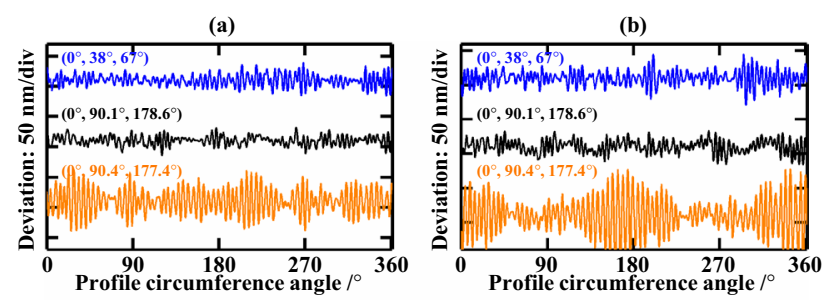

(c)
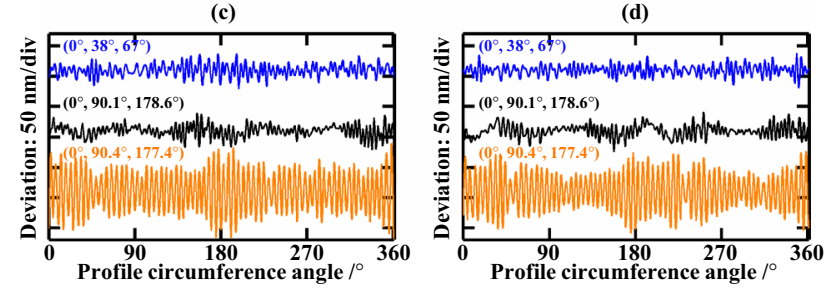

Fig. 4 Profile reconstruction errors with different orientation-angle combinations

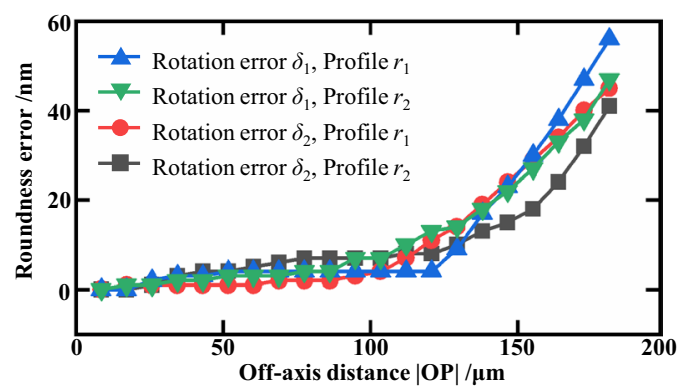

Fig. 5 Roundness error at different eccentricity distances

Figure $6 \mathrm{c}$ shows the experimental setup for the roundness measurement of a cylindrical workpiece. Three selfdesigned chromatic confocal sensors [29] were used to achieve the displacement changes of the rotating workpiece surface within $400 \mu \mathrm{m}$. The axial resolution of the chromatic confocal sensors is approximately $0.1 \mu \mathrm{m}$ in the full measurement range. Before the measurement, three probes were well adjusted to stay on the same horizontal plane and intersect at one point. Then, the workpiece was mounted on the rotary platform with a small eccentricity distance. The biggest rotation error of the rotary platform from Sanying Motion Control Instruments Ltd. was approximately $5 \mu \mathrm{m}$. The outer diameter of the workpiece is approximately $38 \mathrm{~mm}$, and the probe's diameter is approximately $30 \mathrm{~mm}$. The probe's working distance from the probes to the workpiece surface is approximately $7 \mathrm{~mm}$, easily causing

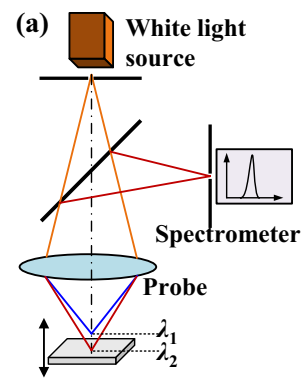

(b)
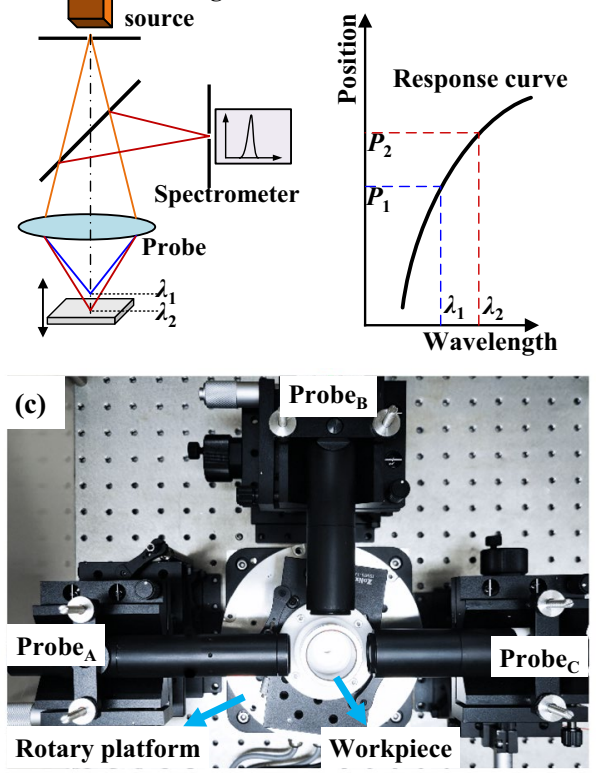

Fig. 6 a Basic principles of a chromatic confocal sensor; b wavelength-position response curve; c experimental setup for the roundness measurement 
interference if the orientation angles are dense, such as $\left(0^{\circ}\right.$, $38^{\circ}$, and $\left.67^{\circ}\right)$. Thus, we set these angles to be $\left(0^{\circ}, 90.1^{\circ}\right.$, and $178.6^{\circ}$ ) in the following experiments.

Based on the experimental setup, the workpiece was rotated for several circles to synchronously obtain the displacement signals in three displacement sensors, as shown in Fig. 7. The signal curves turn along the rotation angle $\theta$ from $0^{\circ}$ to $360^{\circ}$. The floating ranges are all approximately $\pm 10 \mu \mathrm{m}$, indicating a small eccentricity distance to a certain extent. The signal outputs $\left(S_{\mathrm{A}}, S_{\mathrm{C}}\right)$ of Probe $_{\mathrm{A}}$ and Probe $_{\mathrm{C}}$ are almost contrary. In reality, the phase difference of $S_{\mathrm{A}}, S_{\mathrm{B}}$, and $S_{\mathrm{C}}$ can reveal the orientation angles $\alpha$ and $\beta$ accurately. Using six measurements after a careful adjustment, the final orientation angles were determined and equaled as $90.1^{\circ}$ and $178.6^{\circ}$, in accordance with the design target.

Using the error separation technique, six profile functions were derived from the above signals, as shown in Fig. 8. The radius of the workpiece varied within $\pm 2.5 \mu \mathrm{m}$, and the measurement repeatability was less than $0.5 \mu \mathrm{m}$. By equaling six profile functions, the measured profile is plotted in the polar coordinates in Fig. 8b. Furthermore, the roundness was derived using the least squares method as 4.43, 4.18, $4.54,4.89,4.83$, and $4.66 \mu \mathrm{m}$, whose mean is approximately $4.59 \mu \mathrm{m}$. To certify the measurement results, we employed an ultraprecision roundness meter from Kosaka Laboratory Ltd. (EC2500H) to perform a single-probe roundness measurement. The rotation error of the aerostatic spindle was less than $20 \mathrm{~nm}$, and the axial accuracy of the contacting displacement probe was approximately $0.1 \mu \mathrm{m} / 100 \mathrm{~mm}$. The profile of the roundness meter is also plotted in Fig. 8b, where the frequency components at harmonic orders of
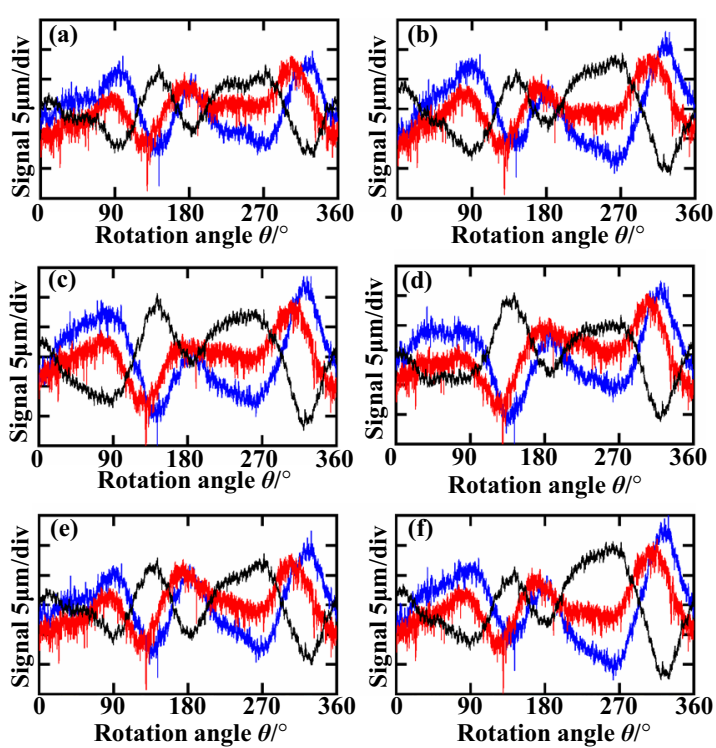

Fig. 7 Output signals in the three displacement probes for six measurements with the orientation-angle combination of $\left(0^{\circ}, 90.1^{\circ}\right.$, and $\left.178.6^{\circ}\right)$
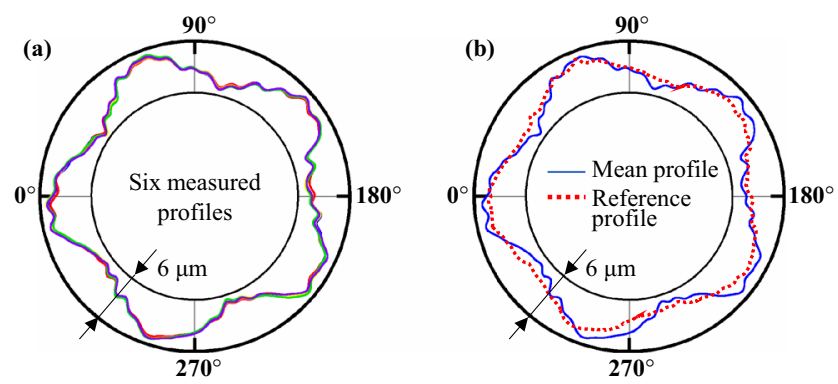

Fig. 8 a Six profiles from the error separation process of Fig. 7; b mean profile and reference profile from an ultraprecise roundness meter

0 and 1 were removed. Based on the results, the two profiles match well with each other within $1 \mu \mathrm{m}$. The roundness from the proposed three-probe experimental system is only $0.03 \mu \mathrm{m}$ smaller than that from the roundness meter of $4.62 \mu \mathrm{m}$.

Another nonoptimal orientation-angle combination $\left(0^{\circ}\right.$, $90.4^{\circ}$, and $177.4^{\circ}$ ) was also tested, whose orientation angle $\beta$ is out of the optimized angle range $\left(178.4-179^{\circ}\right)$ in the simulation. The displacement signals, whose varying ranges are also $\pm 10 \mu \mathrm{m}$, are shown in Fig. 9.

In the same way, six workpiece profiles can be derived through error separation with the displacement signals and they are shown in Fig. 10a. The average of these profiles is plotted in the polar coordinate with the comparison of the measurement results using the ultraprecision roundness meter EC2500H in Fig. 10b. Using the least squares method,
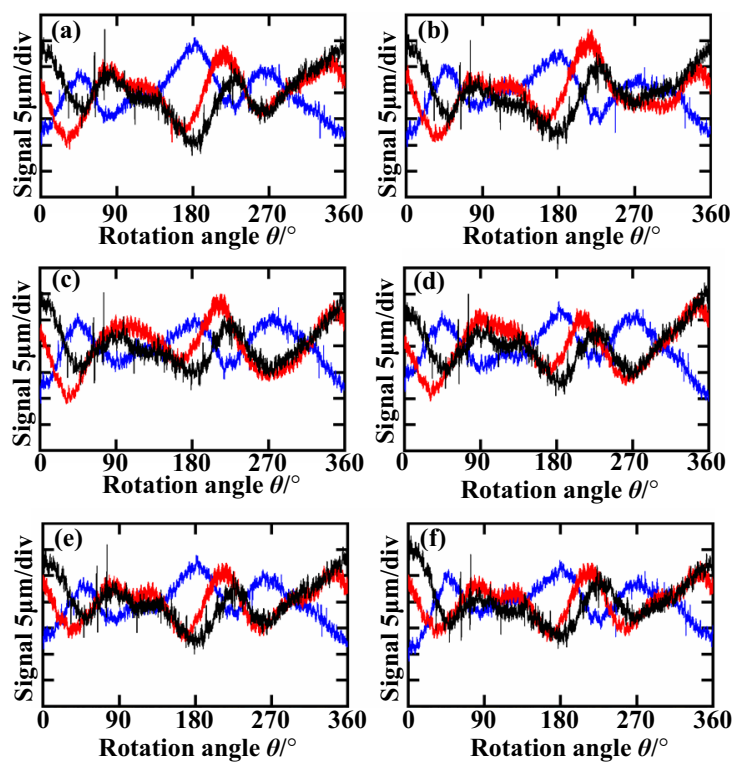

Fig. 9 Output signals in the three displacement probes for six measurements with the orientation-angle combination of $\left(0^{\circ}, 90.4^{\circ}\right.$, and $\left.177.4^{\circ}\right)$ 

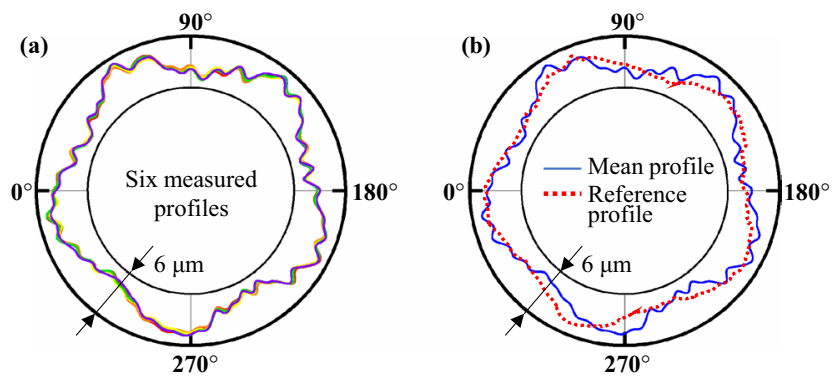

Fig. 10 a Six profiles from the error separation process of Fig. 9; b mean profile and reference profile from the ultraprecise roundness meter

the obtained average roundness from the three-probe method was approximately $4.15 \mu \mathrm{m}$, approximately $0.47 \mu \mathrm{m}$ smaller than that from the roundness meter of $4.62 \mu \mathrm{m}$.

In conclusion, the measurement result with the orientation-angle combination $\left(0^{\circ}, 90.4^{\circ}\right.$, and $\left.177.4^{\circ}\right)$ is worse than the proposed combination $\left(0^{\circ}, 90.1^{\circ}\right.$, and $\left.178.6^{\circ}\right)$. Except for the adjustment uncertainty, the harmonic suppression could be the main reason for the measurement difference. According to Eq. (12), the smallest harmonic order $\omega_{s}$ except 1 is 239 for $\left(0^{\circ}, 90.1^{\circ}\right.$, and $\left.178.6^{\circ}\right)$, bigger than 119 for $\left(0^{\circ}\right.$, $90.4^{\circ}$, and $177.4^{\circ}$ ). Hence, the former combination provides more information on the profile than the latter one. Through comparison, we found that the proposed combination $\left(0^{\circ}\right.$, $90.1^{\circ}$, and $178.6^{\circ}$ ) is indeed the proper choice for the threeprobe error separation.

Furthermore, we explored the influence of the eccentricity distance on the roundness measurement. As shown in Fig. 11a, c, and e, the output signals vary with increasing peak-valley ranges of approximately $24.3,48.4$, and $125.3 \mu \mathrm{m}$. If the rotation error and profile are constant in the rotation, then the displacement output obtains its maximum or minimum when $O P$ is collinear with the optical axis of the chromatic confocal sensor. Thus, the peak-valley range is approximately twice the eccentricity distance $|O P|$. That is, the eccentricity distance increases from approximately 12 , 24, and $63 \mu \mathrm{m}$ in Fig. 11a, c, and e. According to the threeprobe error separation, the roundness is derived as 4.66, 5.07, and $5.18 \mu \mathrm{m}$ in Fig. 11b, d, and f. Evidently, the last two results are bigger than $4.62 \mu \mathrm{m}$ of the ultraprecision roundness meter. The roundness trend is in accordance with the simulation results presented in Fig. 5, which show that the roundness error is improved as the eccentricity distance decreases.

\section{Conclusions}

In this study, we examine the three-probe error separation for the roundness measurement. Based on the theoretical analysis, the orientation angles between displacement
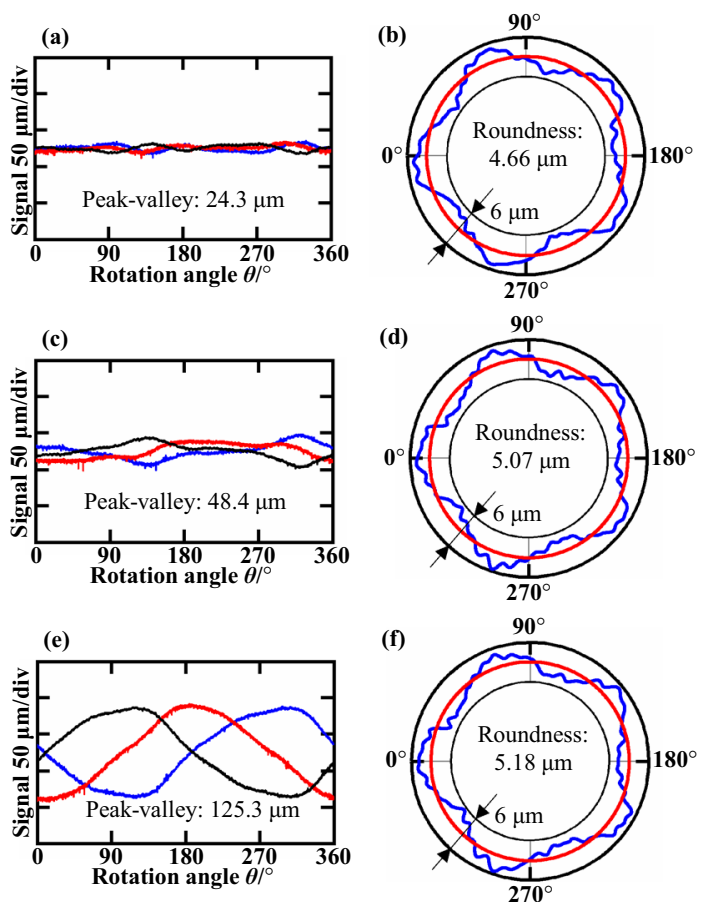

Fig. 11 (a, c, e) Output signals in three probes with different eccentricity distances; $(\mathbf{b}, \mathbf{d}, \mathbf{f})$ the reconstructed profiles based on the output signals

sensors play an important role in harmonic suppression. In detail, we derived harmonic orders to remove invalid frequency components in the profile reconstruction. Then, the Monte Carlo simulation was performed to test the influence of different orientation-angle combinations and eccentricity distances. The optimized orientation-angle combination of $\left(0^{\circ}, 90.1^{\circ}\right.$, and $\left.178.6^{\circ}\right)$ was chosen and tested to avoid the interference among the three probes, showing a similar profile reconstruction accuracy with a traditional selection of $\left(0^{\circ}, 38^{\circ}\right.$, and $\left.67^{\circ}\right)$. Furthermore, the experimental setup employed three similar chromatic confocal displacement sensors to monitor the surface of the cylinder workpiece on the rotary platform. The output signals were used to perform error separation for reconstructing the profile. The measured roundness has a good repeatability of approximately $\pm 0.35 \mu \mathrm{m}$ with an average roundness of $4.59 \mu \mathrm{m}$. By contrast, the nonoptimized orientation-angle combination of $\left(0,90.4^{\circ}\right.$, and $\left.177.4^{\circ}\right)$ was tested and showed an average roundness of $4.15 \mu \mathrm{m}$. In fact, the roundness was measured as $4.62 \mu \mathrm{m}$ by an ultraprecise roundness meter. Hence, the optimized orientationangle combination of $\left(0^{\circ}, 90.1^{\circ}\right.$, and $\left.178.6^{\circ}\right)$ provides a measurement error of only $0.03 \mu \mathrm{m}(0.7 \%)$, much smaller than that from $\left(0^{\circ}, 90.4^{\circ}\right.$, and $\left.177.4^{\circ}\right)$ by $0.47 \mu \mathrm{m}(10.2 \%)$. The comparison clearly shows the feasibility of the proposed orientation-angle combination. Lastly, the influence 
of the eccentricity distance was explored to prove that the roundness error decreases as the eccentricity distance decreases.

In conclusion, this paper provides a useful roundness measurement configuration of the three-probe error separation with the Monte Carlo simulation and actual measurement system. The simulation can provide a reliable reference to optimize measurement parameters and processing algorithms. Moreover, the roundness measurement system was used successfully for a cylindrical workpiece using the optimized orientation-angle combination of $\left(0^{\circ}, 90.1^{\circ}\right.$, and $178.6^{\circ}$ ) with an ordinary low-cost rotary platform.

Acknowledgements This work was supported by the National Natural Science Foundation of China (61905129), Start-up Funding of Tsinghua Shenzhen International Graduate School, Tsinghua University (QD2020001N), Shenzhen Stable Supporting Program (WDZC20200820200655001).

\section{Declarations}

Conflicts of interest The authors declare that they have no conflicts of interest.

Open Access This article is licensed under a Creative Commons Attribution 4.0 International License, which permits use, sharing, adaptation, distribution and reproduction in any medium or format, as long as you give appropriate credit to the original author(s) and the source, provide a link to the Creative Commons licence, and indicate if changes were made. The images or other third party material in this article are included in the article's Creative Commons licence, unless indicated otherwise in a credit line to the material. If material is not included in the article's Creative Commons licence and your intended use is not permitted by statutory regulation or exceeds the permitted use, you will need to obtain permission directly from the copyright holder. To view a copy of this licence, visit http://creativecommons.org/licenses/by/4.0/.

\section{References}

1. Gao W (2010) Precision nanometrology-sensors and measurement systems for nanomanufacturing

2. Bryan J, Clouser R, Holland E (1967) Spindle accuracy. Am Mach 612(25):149-164

3. Ocenasova L, Gapinski B, Cep R, Gregova L, Petrkovska L (2009) Roundness deviation measuring strategy at coordination measuring machines and conventional machines. Proc World Acad Sci Eng Technol 32:523-526

4. Li Q, Shimizu Y, Saito T, Matsukuma H, Gao W (2020) Measurement uncertainty analysis of a stitching linear-scan method for the evaluation of roundness of small cylinders. Appl Sci 10(14):4750

5. Adamczak S, Zmarzy P, Kozior T, Gogolewski D (2017) Assessment of roundness and waviness deviations of elements produced by selective laser sintering technology. In: 23rd international conference engineering mechanics, pp. 70-73

6. Sun C, Wang L, Tan J, Zhao B, Zhou T, Kuang Y (2016) A highaccuracy roundness measurement for cylindrical components by a morphological filter considering eccentricity, probe offset, tip head radius and tilt error. Meas Sci Technol 27:0850088
7. Castro HFF (2008) A method for evaluating spindle rotation errors of machine tools using a laser interferometer. Measurement 41(5):526-537

8. Li X, Shi Z (2009) The relationship between the minimum zone circle and the maximum inscribed circle and the minimum circumscribed circle. Precis Eng 33(3):284-290

9. Sui W, Zhang D (2012) Four methods for roundness evaluation. Phys Proc 24:2159-2164

10. Jiang Q, Feng HY, Ouyang D, Mesay TD (2006) A roundness evaluation algorithm with reduced fitting uncertainty of CMM measurement data. J Manuf Syst 25(3):184-195

11. Evans CJ, Hocken RJ, Estler WT (1996) Self-calibration: reversal, redundancy, error separation, and absolute testing. CIRP Ann 45(2):617-634

12. Marsh ER, Arneson DA, Martin DL (2010) A comparison of reversal and multiprobe error separation. Precis Eng 34(1):85-91

13. Gao W, Kiyono S, Sugawara T (1997) High-accuracy roundness measurement by a new error separation method. Precis Eng 21(2):123-133

14. Shi S, Zhang H, Qu J, Jin G, Kuschnierz R, Czarske J (2019) Measurement uncertainty propagation in spindle error separation techniques: investigation by means of stochastic spectral method. Int J Mach Tools Manuf 141:36-45

15. Cai Y, Xie B, Ling S, Fan KC (2020) On-line measurement method for diameter and roundness error of balls. Nanomanuf Metrol 3:218-227

16. Donaldson RR (1972) A simple method for separating spindle error from test ball roundness error. Ann Cirp 21(1):125-126

17. Cui H, Lei D, Zhang X, Lan H, Jiang Z, Kong L (2019) Measurement and analysis of the radial motion error of aerostatic ultraprecision spindle. Measurement 137:624-635

18. Grejda R, Marsh E, Vallance R (2005) Techniques for calibrating spindles with nanometer error motion. Precis Eng 29(1):113-123

19. Zhang YH, Zhang GX, Yang SM, Li Z (1997) A multipoint method for spindle error motion measurement. CIRP Ann Manuf Technol 46(1):441-445

20. Tiainen T, Viitala R (2021) Robust optimization of multi-probe roundness measurement probe angles. Measurement 168:108146

21. Ding F, Luo XC, Chang WL, Wang ZJ (2019) In situ measurement of spindle radial and tilt error motions by complementary multiprobe method. Nanomanuf Metrol 2(4):225-234

22. Viitala R, Widmaier T, Hemming B, Tammi K, Kuosmanen $P$ (2018) Uncertainty analysis of phase and amplitude of harmonic components of bearing inner ring four-point roundness measurement. Precis Eng 54:118-130

23. Kato H, Sone RY, Nomura Y (1991) In-situ measuring system of circularity using an industrial robot and a piezoactuator. Bull Jpn Soc Precis Eng 25(2):130-135

24. Cappa S, Reynaerts D, Al-Bender F (2014) A sub-nanometre spindle error motion separation technique. Precis Eng 38(3):458-471

25. Gao W, Kiyono S (1997) On-machine roundness measurement of cylindrical workpieces by the combined three-point method. Meas J Int Meas Confed 21(4):147-156

26. Shi S, Lin J, Wang X, Zhao M (2016) A hybrid three-probe method for measuring the roundness error and the spindle error. Precis Eng 45:403-413

27. Chen Y, Zhao X, Gao W, Hu G, Zhang S, Zhang D (2017) A novel multi-probe method for separating spindle radial error from artifact roundness error. Int J Adv Manuf Technol 93(1-4):623-634

28. Darafon A, Warkentin A, Bauer R (2013) Characterization of grinding wheel topography using a white chromatic sensor. Int $\mathrm{J}$ Mach Tools Manuf 70:22-31

29. Bai J, Li X, Wang X, Wang J, Ni K, Zhou Q (2021) Self-reference dispersion correction for chromatic confocal displacement measurement. Opt Lasers Eng 140:106540 
30. Chen C, Yang W, Wang J, Lu W, Liu X, Jiang X (2019) Accurate and efficient height extraction in chromatic confocal microscopy using corrected fitting of the differential signal. Precis Eng $56: 447-454$

31. Minoni U, Manili G, Bettoni S, Varrenti E, Modotto D, De AC (2013) Chromatic confocal setup for displacement measurement using a supercontinuum light source. Opt Laser Technol 49:91-94

32. Nadim EH, Hichem N, Nabil A, Mohamed D, Olivier G (2014)

Comparison of tactile and chromatic confocal measurements of aspherical lenses for form metrology. Int J Precis Eng Manuf 15(5):821-829

33. Zou X, Zhao X, Li G, Li Z, Sun T (2017) Non-contact onmachine measurement using a chromatic confocal probe for an ultra-precision turning machine. Int $\mathbf{J}$ Adv Manuf Technol 90(5-8):2163-2172 